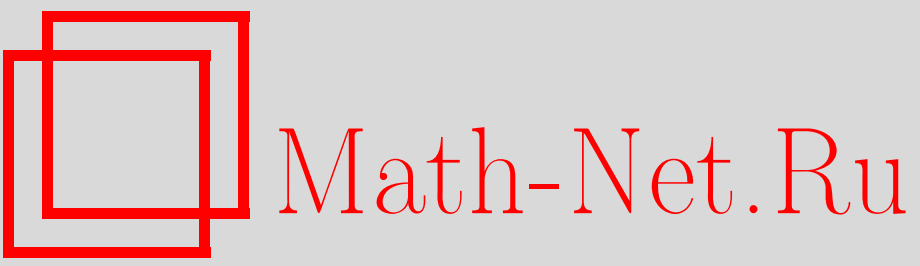

Е. М. Сантанжело, Вычисление энергии Казимира с помощью спектральных функций, ТМФ, 2002, том 131, номер 1, 98-117

DOI: https://doi.org/10.4213/tmf1953

Использование Общероссийского математического портала Math-Net.Ru подразумевает, что вы прочитали и согласны с пользовательским соглашением

http://www.mathnet.ru/rus/agreement

Параметры загрузки:

IP: 54.162 .127 .20

26 апреля 2023 г., 12:34:17 
ТЕОРЕТИЧЕСКАЯ

И МАТЕМАТИЧЕСКАЯ

ФИЗИКА

Том 131, № 1

апрель, 2002

(C) 2002 г.

Е. М. Сантанжело*

\section{ВЫЧИСЛЕНИЕ ЭНЕРГИИ КАЗИМИРА С ПОМОШЬЮ СПЕКТРАЛЬНЫХ ФУНКЦИЙ}

Рассмотрено применение эллиптических дифференциальных операторов и связанных с ними спектральных функций в некоторых задачах квантовой теории поля. Подчеркнута роль дзета-функций и следов ядер теплопроводности при регуляризации энергии Казимира. На простых примерах обсуждается процедура перенормировки.

\section{1. ЭНЕРГИЯ НУЛЕВЫХ КОЛЕБАНИЙ В ПОЛЕВОМ КВАНТОВАНИИ}

В данном разделе вводится понятие энергии Казимира. Рассмотрен простой пример, в котором энергия Казимира вычисляется с помошью двух различных методов регуляризации. Показано, что оба метода взаимосогласованы после перенормировки [1]-[4].

1.1. Свободное массивное нейтральное скалярное поле в неограниченном пространстве-времени Минковского. Рассмотрение проблемы вакуумной энергии (энергии основного состояния) в квантовой теории поля удобно начать с обсуждения простого примера свободного массивного нейтрального скалярного поля в неограниченном пространстве-времени Минковского. Классическая плотность лагранжиана в данном случае имеет вид

$$
\mathcal{L}=\frac{1}{2}(\partial \phi)^{2}-\frac{1}{2} m^{2} \phi^{2}
$$

где $m$ - масса, соответствуюшая полю $\phi$. Из формализма Эйлера-Лагранжа следует классическое уравнение Клейна-Гордона

$$
\left(\partial^{\mu} \partial_{\mu}+m^{2}\right) \phi=0
$$

Канонически-сопряженный данному полю импульс определяется как

$$
\pi=\frac{\partial \mathcal{L}}{\partial\left(\partial_{0} \phi\right)}=\partial_{0} \phi
$$

* Departamento de Física, Facultad de Ciencias Exactas, Universidad Nacional de La Plata, La Plata, Argentina. E-mail: mariel@obelix.fisica.unlp.edu.ar 
так что классический гамильтониан имеет вид

$$
H=\int d^{3} x \frac{1}{2}\left[\pi^{2}+(\nabla \phi)^{2}+m^{2} \phi^{2}\right]
$$

Переход к квантовой теории поля достигается заменой функций $\phi$ и $\pi$ операторнозначными функциями, скобок Пуассона коммутаторами и наложением при равных временах условия

$$
\left[\hat{\phi}(t, \vec{x}), \hat{\pi}\left(t, \overrightarrow{x^{\prime}}\right)\right]=i \delta^{3}\left(\vec{x}-\overrightarrow{x^{\prime}}\right),
$$

правые части остальных коммутаторов равны нулю.

Перейдем к импульсному представлению, где $k^{\mu}=\left(k^{0}, \vec{k}\right)=\left(\omega_{\vec{k}}, \vec{k}\right), \quad \omega_{\vec{k}}=\omega_{-\vec{k}}=$ $\sqrt{\vec{k}^{2}+m^{2}}>0$, и оба поля могут быть разложены следующим образом:

$$
\begin{aligned}
& \hat{\phi}(t, \vec{x})=\int d \tilde{k}\left[\hat{a}(\vec{k}) e^{-i k x}+\hat{a}^{\dagger}(\vec{k}) e^{i k x}\right] \\
& \hat{\pi}(t, \vec{x})=-i \int d \tilde{k}\left[\hat{a}(\vec{k}) e^{-i k x}-\hat{a}^{\dagger}(\vec{k}) e^{i k x}\right]=\partial_{0} \hat{\phi}(t, \vec{x}) .
\end{aligned}
$$

Здесь

$$
d \tilde{k}=\frac{d^{3} k}{(2 \pi)^{3} 2 \omega_{\vec{k}}}
$$

- лоренц-инвариантная мера. Следует обратить внимание на то, что величины $\omega_{\vec{k}}$ являются квадратными корнями из собственных значений оператора $\Delta+m^{2}$.

Условие квантования (1) записывается в виде

$$
\left[\hat{a}(\vec{k}), \hat{a}^{\dagger}\left(\overrightarrow{k^{\prime}}\right)\right]=(2 \pi)^{3} 2 \omega_{k} \delta^{3}\left(\vec{k}-\overrightarrow{k^{\prime}}\right)
$$

Правые части всех остальных коммутаторов равны нулю. Оператор Гамильтона принимает вид

$$
\begin{aligned}
\widehat{H} & =\frac{1}{2} \int d \tilde{k} \omega_{\vec{k}}\left[\hat{a}^{\dagger}(\vec{k}) \hat{a}(\vec{k})+\hat{a}(\vec{k}) \hat{a}^{\dagger}(\vec{k})\right]= \\
& =\frac{1}{2} \int d \tilde{k} \omega_{\vec{k}}\left[2 \hat{a}^{\dagger}(\vec{k}) \hat{a}(\vec{k})+(2 \pi)^{3} 2 \omega_{\vec{k}} \delta^{3}(0)\right] .
\end{aligned}
$$

Определим вакуумное состояние условиями

$$
\hat{a}(\vec{k})|0\rangle=0, \quad\langle 0 \mid 0\rangle=1, \quad\langle 0|\widehat{H}| 0\rangle=\frac{1}{2} \int d^{3} k \omega_{\vec{k}} \delta^{3}(0) .
$$

Множитель $\delta^{3}(0)$ в приведенных формулах не имеет математического смысла и появляется из-за бесконечного размера системы. Чтобы придать смысл последнему выражению, рассматриваемую систему помещают в куб объема $V$, накладывают периодические граничные условия на поле и затем устремляют объем к бесконечному пределу. Тогда

$$
\langle 0|\widehat{H}| 0\rangle=\frac{V}{2} \int \frac{d^{3} k}{(2 \pi)^{3}} \sqrt{\vec{k}^{2}+m^{2}},
$$


где $V$ - (бесконечный) объем пространства. Из последнего выражения видно, почему энергия нулевых колебаний на единицу объема расходится в случае свободного скалярного поля: расходимость появляется из-за суммы энергий нулевых колебаний бесконечного числа осцилляторов. Вводя нормальное упорядочение, можно положить вакуумное значение энергии для свободной теории в неограниченном пространстве Минковского равным нулю. Но тогда возникает естественный вопрос: каково значение вакуумной энергии в присутствии фонового поля и/или когда квантованное поле занимает ограниченную область пространства и, таким образом, зависит от граничных условий?

Энергия Казимира [2] - это вакуумная энергия, вычисленная при наличии дополнительных условий, упомянутых в приведенном вопросе.

1.2. Безмассовое скалярное поле, удовлетворяющее граничным условиям Дирихле в одном пространственном измерении. Допустим, что поле заключено между двумя параллельными пластинами, разделенными расстоянием $a$ в направлении $x$. Поле удовлетворяет граничным условиям на пластинах

$$
\phi(t, 0, y, z)=\phi(t, a, y, z)=0 .
$$

В этом случае отрицательно- и положительно-частотные компоненты поля пропорциональны $\sin k_{n} x$, где $k_{n}=n \pi / a, n=1,2, \ldots$, и $\omega_{n}=\left(k_{n}^{2}+k_{y}^{2}+k_{z}^{2}\right)^{1 / 2}$. Таким образом, вакуумная энергия на единицу плошади пластин дается выражением

$$
\frac{E_{V}}{A}=\frac{1}{2} \int_{-\infty}^{\infty} \frac{d k_{y} d k_{z}}{(2 \pi)^{2}} \sum_{n=1}^{\infty}\left(\left(\frac{n \pi}{a}\right)^{2}+k_{y}^{2}+k_{z}^{2}\right)^{\frac{1}{2}}
$$

Как и ожидалось, эта вакуумная энергия на единицу поперечной площади расходится: расходятся как ряд, так и интеграл. Чтобы интерпретировать данное выражение для энергии, следует, как обычно, регуляризовать его, изолировать расходимости и затем ренормировать (если возможно) классическую энергию исходя из физических предположений.

Первый метод, который мы применим, известен как регуляризация на основе дзета-функции [5], [6]. Он основывается на аналитических свойствах дзета-функции оператора, который в данном случае есть минус лапласиан. Формальное определение спектральной функции, известной как дзета-функция, будет дано в следующем разделе.

Определим величину ${ }^{1)}$

$$
\frac{E_{V}}{A}=\left.\frac{\mu}{2} \int_{-\infty}^{\infty} \frac{d k_{y} d k_{z}}{(2 \pi)^{2}} \sum_{n=1}^{\infty}\left(\left(\frac{n \pi}{a \mu}\right)^{2}+\left(\frac{k_{y}}{\mu}\right)^{2}+\left(\frac{k_{z}}{\mu}\right)^{2}\right)^{-\frac{s}{2}}\right|_{s=-1}
$$

Здесь $s$ - комплексная переменная с достаточно большой вешественной частью $\operatorname{Re}(s)$, чтобы обеспечить сходимость. В этой области рассматриваемое выражение будет определять аналитическую по $s$ функцию. Вакуумная энергия определяется через аналитическое продолжение функции к точке $s=-1$. Параметр $\mu$ размерности массы введен,

\footnotetext{
1) Для простоты мы сохраняем то же обозначение и для перенормированной величины.
} 
ВЫЧИСЛЕНИЕ ЭНЕРГИИ КАЗИМИРА С ПОМОЩЬЮ СПЕКТРАЛЬНЫХ ФУНКЦИЙ 101

чтобы сделать выражение под знаком суммы в (2) безразмерным. Он должен исчезать из любого физически осмысленного результата.

Теперь, использовав представление [7]

$$
z^{-s}=\frac{1}{\Gamma(s)} \int_{0}^{\infty} d t t^{s-1} e^{-z t}, \quad \operatorname{Re} s>0, \quad \operatorname{Re} z>0
$$

уравнение (2) можно переписать в виде

$$
\begin{aligned}
\frac{E_{V}}{A}= & \frac{\mu}{2} \int_{-\infty}^{\infty} \frac{d k_{y} d k_{z}}{(2 \pi)^{2}} \sum_{n=1}^{\infty} \frac{1}{\Gamma\left(\frac{s}{2}\right)} \int_{0}^{\infty} d t t^{\frac{s}{2}-1} \times \\
& \times\left.\exp \left\{\left(\left(\frac{n \pi}{a \mu}\right)^{2}+\left(\frac{k_{y}}{\mu}\right)^{2}+\left(\frac{k_{z}}{\mu}\right)^{2}\right) t\right\}\right|_{s=-1}
\end{aligned}
$$

Для достаточно больших Re $s$ сумму и интеграл можно поменять местами. Тогда

$$
\begin{aligned}
\frac{E_{V}}{A}= & \frac{\mu}{2} \sum_{n=1}^{\infty} \frac{1}{\Gamma\left(\frac{s}{2}\right)} \int_{0}^{\infty} d t t^{\frac{s}{2}-1} e^{-\left(\frac{n \pi}{a \mu}\right)^{2} t} \int_{-\infty}^{\infty} \frac{d k_{y} d k_{z}}{(2 \pi)^{2}} \times \\
& \times\left.\exp \left\{-\left[\left(\frac{k_{y}}{\mu}\right)^{2}+\left(\frac{k_{z}}{\mu}\right)^{2}\right] t\right\}\right|_{s=-1} .
\end{aligned}
$$

Оба гауссовых интеграла в последнем выражении вычисляются. Используя опять представление (3), можно получить

$$
\frac{E_{V}}{A}=\left.\frac{\mu^{3}}{8 \pi} \frac{\Gamma\left(\frac{s}{2}-1\right)}{\Gamma\left(\frac{s}{2}\right)} \sum_{n=1}^{\infty}\left(\frac{n \pi}{a \mu}\right)^{-s+2}\right|_{s=-1}=\left.\frac{\mu^{3}}{4 \pi(s-2)}\left(\frac{a \mu}{\pi}\right)^{s-2} \zeta_{\mathrm{R}}(s-2)\right|_{s=-1}
$$

где использовано определение дзета-функции Римана

$$
\zeta_{\mathrm{R}}(s)=\sum_{n=1}^{\infty} n^{-s}, \quad \operatorname{Re} s>1
$$

Последний ряд определяет аналитическую функцию при $\operatorname{Re} s>1$, а ее аналитическое продолжение на всю $s$-плоскость имеет всего один простой полюс при $s=1$. В частности, дзета-функция при $s=-3$ равняется $1 / 120$.

Таким образом, получаем окончательный результат для вакуумной энергии

$$
\frac{E_{V}}{A}=-\frac{\pi^{2}}{1440 a^{3}}
$$

Регуляризация с помошью дзета-функции в данном простом случае дает конечный результат, дальнейшей перенормировки не требуется. 
Теперь сравним результат (4) с результатом, полученным другим методом регуляризации - методом экспоненциального обрезания. Как мы увидим позже, этот метод основан на использовании другой спектральной функции, известной как след ядра теплопроводности. Определим

$$
\begin{aligned}
\frac{E_{V}}{A}= & \frac{1}{2} \int_{-\infty}^{\infty} \frac{d k_{y} d k_{z}}{(2 \pi)^{2}} \sum_{n=1}^{\infty}\left(\left(\frac{n \pi}{a \mu}\right)^{2}+\left(\frac{k_{y}}{\mu}\right)^{2}+\left(\frac{k_{z}}{\mu}\right)^{2}\right)^{\frac{1}{2}} \times \\
& \times\left.\exp \left\{-\left(\left(\frac{n \pi}{a \mu}\right)^{2}+\left(\frac{k_{y}}{\mu}\right)^{2}+\left(\frac{k_{z}}{\mu}\right)^{2}\right)^{\frac{1}{2}} t\right\}\right|_{t=0} .
\end{aligned}
$$

Экспонента вводится для того, чтобы обеспечить сходимость, давая таким образом возможность переставить сумму и интеграл. Параметр $\mu$ размерности массы снова произволен. Последнее соотношение может быть также записано в виде

$$
\begin{aligned}
\frac{E_{V}}{A} & =-\left.\frac{\mu}{2} \frac{d}{d t} \int_{-\infty}^{\infty} \frac{d k_{y} d k_{z}}{(2 \pi)^{2}} \sum_{n=1}^{\infty} \exp \left\{-\left(\left(\frac{n \pi}{a \mu}\right)^{2}+\left(\frac{k_{y}}{\mu}\right)^{2}+\left(\frac{k_{z}}{\mu}\right)^{2}\right)^{\frac{1}{2}} t\right\}\right|_{t=0}= \\
& =-\left.\frac{\mu}{4 \pi} \frac{d}{d t} \int_{0}^{\infty} d k k \sum_{n=1}^{\infty} \exp \left\{-\left(\left(\frac{n \pi}{a \mu}\right)^{2}+\left(\frac{k}{\mu}\right)^{2}\right)^{\frac{1}{2}} t\right\}\right|_{t=0} .
\end{aligned}
$$

Меняя местами сумму и интеграл и делая замену переменных, получим

$$
\frac{E_{V}}{A}=-\left.\frac{\mu^{3}}{2} \frac{d}{d t} \sum_{n=1}^{\infty} \int_{\left(\frac{n \pi}{a \mu}\right)^{2}}^{\infty} d k e^{-k^{\frac{1}{2}} t}\right|_{t=0} .
$$

Теперь сумма может быть вычислена с помошю формулы Эйлера-Маклорена

$$
\sum_{n=1}^{\infty} f(n)=-\frac{1}{2} f(0)+\int_{0}^{\infty} f(x) d x-\sum_{k=1}^{\infty} \frac{1}{(2 k) !} B_{2 k} f^{(2 k-1)}(0) .
$$

Можно показать, что окончательный результат для вакуумной энергии в данной схеме регуляризации дается выражением

$$
\frac{E_{V}}{A}=\left.\frac{3 a \mu^{4}}{2 \pi^{2} t^{4}}\right|_{t=0}-\left.\frac{\mu^{3}}{4 \pi t^{3}}\right|_{t=0}-\frac{\pi^{2}}{1440 a^{3}} .
$$

Обратим внимание, что в формуле (6) остаются две расходимости в виде полюсов. Первый расходяшийся член в (6) есть вакуумная энергия всего пространства (она получается из интеграла в уравнении (5)). Вторая расходимость связана с модой $n=0$ (из первого члена в правой части того же уравнения) и, таким образом, возникает из-за граничных условий. Обе расходимости могут быть исключены с помощью условия $E_{V} / A \rightarrow 0$, когда $a \rightarrow \infty$. Это условие можно рассматривать как перенормировку классической энергии, которая в силу геометрии данной задачи имеет вид

$$
E_{\text {class }}=p a A+\sigma A,
$$

где $p$ - давление, $\sigma$ - поверхностное натяжение. Остающаяся конечная часть в (6) находится в согласии с результатом дзета-регуляризации (4). Мы рассмотрели простой пример (безмассовое поле и плоские границы). Далее мы изучим взаимосвязь между этими двумя методами регуляризации и обсудим перенормировку энергии Казимира в более общих случаях. 
ВЫЧИСЛЕНИЕ ЭНЕРГИИ КАЗИМИРА С ПОМОЩЬЮ СПЕКТРАЛЬНЫХ ФУНКЦИЙ 103

\section{2. ЭЛЛИПТИЧЕСКИЕ ДИФФЕРЕНЦИАЛЬНЫЕ ОПЕРАТОРЫ И ГРАНИЧНЫЕ ЗАДАЧИ. СПЕКТРАЛЬНЫЕ ФУНКЦИИ}

В предыдушем разделе была вычислена энергия нулевых колебаний с помошњю двух различных методов регуляризации: на основе дзета-функции и экспоненциального обрезания. Они основаны на использовании определенных функций от спектра данного дифференциального оператора, называемых спектральными функциями. В данном разделе рассматриваются условия, при которых эти функции могут быть определены, и некоторые их свойства [8]-[12].

2.1. Дифференциальные операторы на компактных многообразиях без границы. Пусть $M$ - компактное многообразие без границы размерности $n, E$ - комплексное векторное расслоение на $M$.

Дифференииальный оператор с частными производнымми порядка $m$, действующий на слои $E$, может быть записан в локальных координатах в виде

$$
A=\sum_{|\alpha| \leqslant m} a_{\alpha}(x) D_{x}^{\alpha}, \quad D_{x}^{\alpha}=\prod_{j=1}^{n}\left(-i \frac{\partial}{\partial x_{j}}\right)^{\alpha_{j}}, \quad|\alpha|=\sum_{j=1}^{n} \alpha_{j} .
$$

Коэффициенты $a_{\alpha}(x)$ являются в общем случае $(q \times q)$-матрицами.

В качестве примера рассмотрим оператор

$$
-\frac{d^{2}}{d x^{2}}+x \frac{d}{d x}+1
$$

В этом случае $n=1, m=2$, т.е. $j=1,|\alpha|=\alpha_{1} \leqslant 2$. Оператор может быть записан в компактной форме в виде

$$
\sum_{\alpha_{1} \leqslant 2} a_{\alpha_{1}}(x)(-i)^{\alpha_{1}}\left(\frac{d}{d x_{1}}\right)^{\alpha_{1}}
$$

Коэффициенту $a_{0}(x)=1$ соответствует $\alpha_{1}=0 ;$ коэффициент, соответствуюший $\alpha_{1}=1$, есть $a_{1}(x)=i x$, а коэффициенту $a_{2}(x)=1$ соответствует $\alpha_{1}=2$.

Символ оператора $A$ определяется как

$$
\sigma(A)=\sigma(x, \xi)=\sum_{|\alpha| \leqslant m} a_{\alpha}(x) \xi^{\alpha}
$$

Это полином порядка $m$ по дуальной переменной $\xi$, получаемый формальной заменой $D_{x}^{\alpha}$ мономом $\xi^{\alpha}$. Действие оператора на функцию из области его определения может быть записано с помощью символа этого оператора в виде

$$
A f(x)=\int d \xi e^{i x \xi} \sigma(x, \xi) \tilde{f}(\xi)
$$

где $\tilde{f}(\xi)$ - фурье-образ функции $f(x)$. 
Для оператора из предыдушего примера $\sigma(x, \xi)=\xi^{2}+i x \xi+1$. Легко показать, что

$$
\left(-\frac{d^{2}}{d x^{2}}+x \frac{d}{d x}+1\right) f(x)=\int d \xi e^{i x \xi}\left(\xi^{2}+i x \xi+1\right) \tilde{f}(\xi) .
$$

Главный символ - это часть символа с наиболее высоким порядком. Он является однородным полиномом степени $m$ по $\xi$,

$$
\sigma_{m}(A)=\sigma_{m}(x, \xi)=\sum_{|\alpha|=m} a_{\alpha}(x) \xi^{\alpha} .
$$

В нашем примере $\sigma_{2}(x, \xi)=\xi^{2}$.

Говорят, что дифференциальный оператор әллиптичен, если его главный символ обратим при $|\xi|=1$ (отсутствует нулевое собственное значение при $|\xi|=1$ или, что эквивалентно, $\operatorname{det} \sigma_{m}(x, \xi) \neq 0$ при $\left.|\xi|=1\right)$.

В нашем примере эллиптичность оператора очевидна.

Для оператора $A$ его резольвентой является оператор $(A-\lambda I)^{-1}$. Луч $\mathcal{K}=\{\arg \lambda=$ $\theta\}$ в комплексной плоскости $\lambda$ назьвается лучом минимального роста резольвенты, если на таком луче нет собственных значений главного символа, т.е. уравнение

$$
\sigma_{m}(x, \xi) u=\lambda u
$$

имеет только тривиальное решение для $\lambda \in \mathcal{K}$. Можно показать, что вдоль такого луча $L^{2}$-норма резольвенты есть $O\left(|\lambda|^{-1}\right)$. В нашем примере уравнение $\xi^{2} u=\lambda u$ имеет только тривиальное решение для любого $\lambda \neq \xi^{2}$. Так как число $\xi$ вешественно, любой луч в $\mathbb{C}-\mathbb{R}_{+}$является лучом минимального роста.

2.2. Комплексные степени дифференциального оператора. Для эллиптического дифференциального оператора $A$ с лучом минимального роста $\mathcal{K}_{\text {и }} \operatorname{Re} s>0$ можно определить оператор

$$
A^{-s}=\frac{i}{2 \pi} \int_{\Gamma} \lambda^{-s}(A-\lambda I)^{-1} d \lambda,
$$

где кривая $\Gamma$ начинается на $\infty$, подходит вдоль луча $\mathcal{K}$ к малому кругу в начале координат и далее идет обратно на $\infty$ вдоль луча. Заметим, что такая кривая обходит собственные значения главного символа по часовой стрелке.

Для описания оператора $A^{-s}$ можно построить аппроксимацию $B(\lambda)$ резольвенты $(A-\lambda I)^{-1}$, известную как параметрикс. Параметрикс воспроизводит поведение резольвенты при $\lambda \rightarrow \infty$ вдоль луча минимального роста [8].

Если рассматривать $\lambda$ как часть главного символа оператора $A$, параметрикс можно найти в виде

$$
\sigma(B) \sim \sum_{j=0}^{\infty} b_{-m-j}(x, \xi, \lambda)
$$


ВЫЧИСЛЕНИЕ ЭНЕРГИИ КАЗИМИРА С ПОМОЩЬЮ СПЕКТРАЛЬНЫХ ФУНКЦИЙ 105

при налагаемом условии

$$
\sigma(B(A-\lambda I))=I
$$

Коэффициенты $b_{-m-j}$ известны как коэффициенты Сили. Можно проверить (с помощью формулы для символа композиции операторов), что они удовлетворяют следующему набору алгебраических уравнений:

$$
\begin{gathered}
b_{-m}\left(a_{m}-\lambda\right)=1, \\
b_{-m-l}\left(a_{m}-\lambda\right)+\sum\left(\partial_{\xi}^{\alpha} b_{-m-j}\right)\left(D_{x}^{\alpha} a_{m-k}\right)=0, \quad l>0 .
\end{gathered}
$$

Здесь сумма должна быть взята по всем $k+j+|\alpha|=l$ и $j<l, a_{m-k}$ - символы различных порядков дифференциального оператора $A$. Аппроксимация символа оператора $A^{-s}$ дается выражением

$$
\sigma\left(A^{-s}\right) \sim \sum_{j=0}^{\infty} \frac{i}{2 \pi} \int_{\Gamma} \lambda^{-s} b_{-m-j}(x, \xi, \lambda) d \lambda
$$

Исходя из этого выражения, можно показать, что для $\operatorname{Re} m s>n$ ядро $K_{-s}(x, y)$ оператора $A^{-s}$ непрерывно. Для $x \neq y$ оно продолжается на всю плоскость $s$. Для $x=y$ его можно аналитически продолжить до мероморфной функции, единственные сингулярности которой - простые полюсы в точках $s=(n-j) / m, j=0,1 \ldots$ Каждый полюс возникает из-за определенного члена в предыдушем выражении, и вычеты, таким образом, определяются интегралами коэффициентов Сили вдоль контура Г.

2.3. Дзета-функция. Связь с собственными значениями. Первой функцией, которую мы рассмотрим для заданного эллиптического оператора $A$, будет дзета-функция, определенная как след:

$$
\zeta(A, s)=\operatorname{tr} A^{-s}
$$

Аналитические свойства дзета-функции получаются из свойств ядра $K_{-s}(x, x)$. Вычеты в полюсах функции $\zeta(A, s)$ являются интегралами по многообразию $M$ от вычетов, соответствующих $K_{-s}(x, x)$, и, следовательно, определяются коэффициентами Сили. Когда оператор обладает полным ортогональным набором собственных функций, его дзета-функция может быть выражена через соответствующие собственные значения.

Предположим, что на расслоении гладко определено эрмитово скалярное произведение и элемент объема $d v$ гладко определен на $M$. Если оператор $A$ нормален по отношению к этим структурам $\left(A^{\dagger} A=A A^{\dagger}\right)$, то он обладает полным ортонормированным набором собственных функций $A \phi_{k}=\lambda_{k} \phi_{k}$, и можно записать

$$
K_{-s}(x, y) d v_{y}=\sum_{k} \lambda_{k}^{-s} \phi_{k}(x) \phi_{k}^{\dagger}(y) d v_{y}
$$


Полагая $x=y$ и интегрируя по $M$, имеем

$$
\zeta(A, s)=\operatorname{tr} A^{-s}=\sum_{k} \lambda_{k}^{-s}
$$

Рассмотрим в качестве примера на единичном круге оператор

$$
A=-\frac{d^{2}}{d x^{2}}+P
$$

где $P$ - проектор на нулевые моды. Собственные значения оператора $A$ суть $\lambda_{n}=n^{2}$ для $n= \pm 1, \pm 2, \ldots$ и $\lambda_{0}=1$ для нулевой моды. Таким образом,

$$
\zeta(A, s)=2 \sum_{n=1}^{\infty}\left(n^{2}\right)^{-s}+1=2 \zeta_{\mathrm{R}}(2 s)+1 .
$$

Известно, что эта дзета-функция Римана аналитична при $\operatorname{Re} 2 s>1$, т.е. $\operatorname{Re} s>$ $1 / 2=n / m$. Ее аналитическое продолжение имеет простой полюс при $2 s=1$.

2.4. Тепловое ядро и его след. Если все собственные значения главного символа принадлежат области $S_{0}:-\pi / 2+\varepsilon<\arg \lambda<\pi / 2-\varepsilon$, то спектр оператора $A$ принадлежит сектору $S_{\alpha}:-\pi / 2+\varepsilon<\arg (\lambda+\alpha)<\pi / 2-\varepsilon$ для некоторого $\alpha>0$ и можно определить оператор

$$
e^{-A t}=\frac{i}{2 \pi} \int_{\Gamma} e^{-\lambda t}(A-\lambda I)^{-1} d \lambda, \quad t>0
$$

где $\Gamma$ - граница $S_{\alpha}$. Можно показать, что $e^{-A t}$ является фундаментальным решением уравнения теплопроводности $A u+\partial u / \partial t=0$ с начальным условием $u(x, 0)=\delta(x)$. Поэтому оператор $e^{-A t}$ назьвается ядром теплопроводности оператора $A$.

Аппроксимация резольвенты $B(\lambda)$ может быть использована в предыдущем интеграле в пределе $t \rightarrow+0$. Таким образом получается асимптотическое разложение для $e^{-A t}$ по увеличивающимся (в обшем случае нецелочисленным) степеням $t$. Коэффициенты в этом разложении также определяются коэффициентами Сили.

Как и ранее, если $A$ обладает полным набором собственных функций, ядро оператора $e^{-A t}$ можно записать в виде

$$
K(t, x, y)=\sum_{k} e^{-\lambda_{k} t} \phi_{k}(x) \phi_{k}^{\dagger}(y) .
$$

След

$$
h(A, t)=\operatorname{tr} e^{-A t}=\sum_{k} e^{-\lambda_{k} t}
$$

является второй спектральной функцией, которую мы будем в дальнейшем использовать.

Сушествует тесная взаимосвязь между дзета-функцией оператора и следом ядра теплопроводности. В самом деле, из соотношений (7) и (8) имеем

$$
\zeta(A, s)=\sum_{k} \lambda_{k}^{-s}=\frac{1}{\Gamma(s)} \int_{0}^{\infty} d z \sum_{k} e^{-\lambda_{k} z} z^{s-1}=\frac{1}{\Gamma(s)} \int_{0}^{\infty} d z h(A, z) z^{s-1},
$$

т.е. эти спектральные функции связаны друг с другом так называемым преобразованием Меллина. 
2.5. Эллиптические граничные системы. Выше мы рассматривали многообразия без границы. Как распространить введенные определения на многообразия с гранищей? Рассмотрим компактное многообразие $M$ размерности $n$ с гладкой границей $\partial M$. Будем обозначать $x=\left(x_{1}, \ldots, x_{n-1}\right)$ координаты на $\partial M$ в каждой локальной координатной системе. Пусть $t \in \mathbb{R}$ - внутренняя нормаль к границе. Таким образом, $(x, t) \in \mathbb{R}^{n}$. Обозначим через $\mathbb{R}_{+}^{n}$ полупространство $t \geqslant 0$. Будем рассматривать в $\mathbb{R}_{+}^{n}$ дифференциальный оператор порядка $m$,

$$
A=\sum_{j=0}^{m} A_{j}(x, t) D_{t}^{m-j}, \quad D_{t}=-i \frac{\partial}{\partial t},
$$

где $A_{j}$ - дифференциальный оператор порядка не выше $j$ на $\mathbb{R}^{n-1}$. Тогда, обозначая через $(\xi, \tau)$ символьные переменные, соответствуюшие $(x, t)$, имеем

$$
\sigma(A)=\sum_{j} \sigma\left(A_{j}\right)(x, t, \xi) \tau^{m-j}
$$

Главный символ

$$
\sigma_{m}=\sum_{j} \sigma_{j}\left(A_{j}\right)(x, t, \xi) \tau^{m-j} .
$$

Кроме того, определим главный символ в частных производных

$$
\sigma_{m}^{\prime}=\sum_{j} \sigma_{j}\left(A_{j}\right)(x, 0, \xi) D_{t}^{m-j}
$$

Предположим, что вблизи границы заданы операторы (определяющие граничные условия)

$$
B_{j}=\sum_{k=1}^{m} B_{j k} D_{t}^{m-k}, \quad 1 \leqslant j \leqslant \frac{m q}{2}
$$

где $B_{j k}$ - система дифференциальных операторов $((1 \times q)$-матриц $)$, действуюших на $\mathbb{R}^{n-1}$. Рассмотрим случай, когда эти граничные операторы просто мультипликативны. Тогда

$$
\sigma\left(B_{j}\right)=\sum_{k=1}^{m} \sigma\left(B_{j k}\right) \tau^{m-k}, \quad \sigma^{\prime}\left(B_{j}\right)=\sum_{k=1}^{m} \sigma\left(B_{j k}\right) D_{t}^{m-k} .
$$

Набор операторов $A, B_{1}, \ldots, B_{m q / 2}$ составляет әллиптическую граничную системy, если $A$ эллиптичен и для произвольного $g=\left(g_{1}, \ldots, g_{m q / 2}\right), x \in \mathbb{R}^{n-1}$ и $\xi \neq 0$, $\xi \in \mathbb{R}^{n-1}$, сушествует единственное решение следуюшей задачи:

$$
\begin{gathered}
\sigma_{m}^{\prime}(A)\left(x, \xi, D_{t}\right) u=0, \quad t>0, \\
\lim _{t \rightarrow \infty} u(t)=0, \\
\sigma^{\prime}\left(B_{j}\right)\left(x, \xi, D_{t}\right) u=g_{j} \text { при } t=0, \quad j=1, \ldots, \frac{m q}{2} .
\end{gathered}
$$


Эти условия на $u(t)$ также известны как условия Лопатинского-Шапиро [12]. Когда они выполняются, можно определить оператор $A_{B}$ как оператор $A$, действуюший на функции $u$ такие, что $B_{j} u=0$.

Набор $A, B_{1}, \ldots, B_{m q / 2}$ составляет сильную әллиптическую граничную систему на конусе $\mathcal{K} \subset \mathbb{C}$, включая начало координат, если

а) для $(\xi, \tau) \neq(0,0) \sigma_{m}(A)$ не имеет собственных значений на $\mathcal{K}$,

б) для любого $x$ и любых $(\xi, \lambda) \neq(0,0)$, где $\lambda \in \mathcal{K}$, граничная задача

$$
\begin{gathered}
\sigma_{m}^{\prime}(A)\left(x, \xi, D_{t}\right) u=\lambda u, \\
\lim _{t \rightarrow \infty} u(t)=0, \\
\sigma^{\prime}\left(B_{j}\right)\left(x, \xi, D_{t}\right) u=g_{j} \text { при } t=0, \quad j=1, \ldots, \frac{m q}{2},
\end{gathered}
$$

имеет единственное решение. Заметим, что эти условия сводятся к условиям Лопатинского-Шапиро для $\lambda=0$. Конус $\mathcal{K}$ известен как конус Агмона [11].

При выполнении условия сильной эллиптичности существует аппроксимация резольвенты $\left(A_{B}-\lambda\right)^{-1}[9]$, с помошью которой можно получить, что

$$
\left(A_{B}\right)^{-s}=\frac{i}{2 \pi} \int_{\Gamma} \lambda^{-s}\left(A_{B}-\lambda I\right)^{-1} d \lambda,
$$

где $\Gamma$ - кривая на конусе, на которой сушествует оператор $\left(A_{B}-\lambda I\right)^{-1}$. Коэффииииенты в разложении параметрикса должны теперь удовлетворять не только условию обратимости оператора $A_{B}-\lambda I$, но и граничным условиям. Тогда кроме объемных коэффициентов Сили $b_{-m-j}$ появляются новые граничные коэффициенты $d_{m-j}$, и их определение приводит к набору дифференциальных (а не алгебраических) уравнений.

Условия, определяюшие структуру полюсов $K_{-s}(x, y)$, похожи на аналогичные условия в случае отсутствия границы. Однако в данном случае вычеты полюсов определяются суммой объемных интегралов коэффициентов $b$ и граничных интегралов коэфффициентов $d$. Дзета-функция может быть, как и ранее, определена как след $(-s)$-й степени. В частности, если оператор $A_{B}$ имеет полный набор собственных функций, то имеют место разложения (7) и

$$
h\left(A_{B}, t\right)=\sum_{k} e^{-\lambda_{k} t} .
$$

Обе спектральные функции (7) и (10) опять связаны преобразованием Меллина.

В качестве примера рассмотрим лапласиан на границе цилиндра с граничными условиями Дирихле. Пусть $A=-\partial_{x}^{2}-\partial_{y}^{2}$ действует на функции $\varphi(x, y)$ такие, что $\varphi(0, y)=$ $\varphi(1, y)=0$ и эти функции периодичны в направлении $y$. При этом граница соответствует точкам $x=0, x=1$. Граничный оператор просто мультипликативен, $B=1$. Обозначим через $(\tau, \xi)$ переменные Фурье, связанные с $(x, y)$. Тогда

$$
\begin{aligned}
& \sigma(A)=\sigma_{2}(A)=\xi^{2}+\tau^{2}, \\
& \sigma(B)=\sigma_{0}(B)=\sigma_{0}^{\prime}(B)=1 .
\end{aligned}
$$


Можно показать, что дифференциальный оператор $A$ эллиптичен. Действительно, $\sigma_{2}(A)=\xi^{2}+\tau^{2} \neq 0$ для $(\tau, \xi) \neq(0,0)$, так как $\tau, \xi \in \mathbb{R}$.

Можно также показать, что граничная задача эллиптична в слабом (по Лопатинскому-Шапиро) смысле. Рассмотрим гранишу $x=0$. Тогда $x$ является переменной, нормальной к границе. Дифференциальное уравнение

$$
\sigma_{2}^{\prime}(A) u=\left(-\partial_{x}^{2}+\xi^{2}\right) u=0
$$

имеет при $\xi \neq 0$ решение вида

$$
u=C e^{|\xi| x}+D e^{-|\xi| x}
$$

Из условия убывания этого решения при $x \rightarrow \infty$ следует, что $C=0$. Тогда задача $u(0, \xi)=g$ имеет при любых $g$ единственное решение $D=g$, которое показывает, что условия слабой эллиптичности выполняются при $x=0$. По аналогии можно показать, что они вьполняются и при $x=1$.

Дифференциальный оператор $A$ имеет конус Агмона. В самом деле, уравнение

$$
\sigma_{2} u=\left(\tau^{2}+\xi^{2}\right) u=\lambda u
$$

с $(\tau, \xi) \neq(0,0)$ имеет нетривиальные решения только при $\lambda=\tau^{2}+\xi^{2} \in \mathbb{R}_{+}$, т.е. дифференциальньй оператор имеет конус Агмона $\mathcal{K}=\mathbb{C}-\mathbb{R}_{+}$.

Можно также показать, что для рассматриваемого примера граничная задача сильно эллиптична (имеет конус Агмона). Действительно, снова рассмотрим границу $x=0$. Дифференциальное уравнение

$$
\sigma_{2}^{\prime}(A) u=\left(-\partial_{x}^{2}+\xi^{2}\right) u=\lambda u
$$

имеет при $(\xi, \lambda) \neq(0,0)$ решение вида

$$
u=C e^{\sqrt{\xi^{2}-\lambda^{2}} x}+D e^{-\sqrt{\xi^{2}-\lambda^{2}} x} .
$$

Из условия убывания решения при $x \rightarrow \infty$ следует, что $C=0$, т.е. задача $u(0, \xi, \lambda)=g$ имеет при любых $g$ единственное решение $D=g$. Отсюда с учетом того, что дифференциальньй оператор $A$ имеет конус Агмона, следует, что граничная задача сильно эллиптична на $\mathcal{K}=\mathbb{C}-\mathbb{R}_{+}$.

\section{3. СРАВНЕНИЕ ДЗЕТА-РЕГУЛЯРИЗАЦИИ И ЭКСПОНЕНЦИАЛЬНОЙ РЕГУЛЯРИЗАЦИИ ЭНЕРГИИ КАЗИМИРА}

В данном разделе показано, что в обшем случае как дзета-регуляризация, так и экспоненциальная регуляризация дают расходящиеся ответы для энергии Казимира. Устанавливается взаимосвязь меж ду этими регуляризациями и обсуждается сушествование единственного, физически осмысленного после перенормировки, результата [13], [14]. 
3.1. Общий результат. В первом разделе мы изучили простой пример вычисления энергии Казимира: безмассовое скалярное поле между "проводящими" пластинами. В этом примере мы получили конечный результат для вакуумной энергии в дзета-регуляризации и полюсную расходимость при экспоненциальном обрезании. Более того, зависимость расходяшихся членов от расстояния между пластинами соответствовала классическому действию и отличалась от зависимости конечной части от расстояния между пластинами. Таким образом, расходимости можно устранить перенормировкой.

Возникает несколько вопросов. Возможна ли перенормировка в более обшем случае? Дает ли дзета-функция всегда конечный ответ? Всегда ли расходимости в экспоненциальной регуляризации присутствуют в виде полюсов? Какова в более общем случае взаимосвязь между результатами обеих регуляризаций?

Чтобы ответить на эти вопросы, будем использовать обшие результаты раздела 2 о структуре дзета-функции и следа ядра теплопроводности, приложенные к операторам второго порядка.

Напомним, что, например, в скалярном случае вакуумная энергия дается формулой

$$
E_{V}=\frac{1}{2} \sum_{n} \omega_{n}
$$

где $\omega_{n}$ - энергии нулевых колебаний. Рассмотрим скалярное поле в $(d+1)$-мерном пространстве-времени, где $d$ - размерность компактного пространственного многообразия с (или без) гладкой границей.

Пусть $\omega_{n}=\lambda_{n}^{1 / 2}$, где $\lambda_{n}$ удовлетворяют соответствуюшей граничной задаче

$$
D_{B} \varphi_{n}=\eta_{n} \varphi_{n}
$$

где

$$
\eta_{n}=\left\{\begin{array}{lll}
\lambda_{n} & \text { при } & D_{B}=D, \\
0 & \text { при } & D_{B}=B .
\end{array}\right.
$$

Здесь $D$ - дифференциальный оператор второго порядка на $M, B$ - оператор, определяюший граничные условия.

Дзета-регуляризованная [5], [6] вакуумная энергия определяется равенствами

$$
\left.E_{\zeta} \equiv \frac{\mu}{2} \sum_{n}\left(\frac{\lambda_{n}}{\mu^{2}}\right)^{-\frac{s}{2}}\right|_{s=-1}=\left.\frac{\mu}{2} \zeta\left(\frac{s}{2}, \frac{D_{B}}{\mu^{2}}\right)\right|_{s=-1}=\left.\frac{\mu}{2} \operatorname{tr}\left(\frac{D_{B}}{\mu^{2}}\right)^{-\frac{s}{2}}\right|_{s=-1} .
$$

Регуляризованное с помошью экспоненциального обрезания выражение дается формулой

$$
\left.E_{\exp } \equiv \frac{\mu}{2} \sum_{n} \frac{\lambda_{n}^{\frac{1}{2}}}{\mu} \exp \left\{-t \frac{\lambda_{n}^{\frac{1}{2}}}{\mu}\right\}\right|_{t=0}=-\left.\frac{\mu}{2} \frac{d}{d t}\left(h\left(t, \frac{D_{B}^{\frac{1}{2}}}{\mu}\right)\right)\right|_{t=0},
$$


где

$$
h\left(t, \frac{D_{B}^{\frac{1}{2}}}{\mu}\right)=\sum_{n} \exp \left\{-t \frac{\lambda_{n}^{\frac{1}{2}}}{\mu}\right\}=\operatorname{tr}\left(\exp \left\{-\frac{t}{\mu} D_{B}^{\frac{1}{2}}\right\}\right) .
$$

Напомним, что $\mu$ в обоих случаях - произвольный параметр размерности массы.

Для того чтобы изучить взаимосвязь между обеими регуляризациями, используем следуюший хорошо известный результат.

Лемма 1 [10]. Пусть D - дифференииальный оператор второго порядка, действующий на гладком компактном d-мерном многообразии $M$, и пусть $B$ - дифференииальный оператор, определяющий граничные условия на дМ. Если граничная задача (12) сильно әллиптична на $\mathbb{C}-\mathbb{R}_{+}$, тогда

а) функиия

$$
\mu^{-s} \Gamma\left(\frac{s}{2}\right) \zeta\left(\frac{s}{2}, \frac{D_{B}}{\mu^{2}}\right)
$$

аналитична при $\operatorname{Re} s>d$ и ее можсн аналитически продолжить до мероморфной функиии со следующей структурой сингулярностей:

$$
\mu^{-s} \Gamma\left(\frac{s}{2}\right) \zeta\left(\frac{s}{2}, \frac{D_{B}}{\mu^{2}}\right)=\sum_{j=0}^{N} \frac{2 a_{j}}{s+j-d}+r_{N}\left(\frac{s}{2}\right)
$$

әде функция $r_{N}(s / 2)$ аналитична для $\operatorname{Re} s>d-N-1$, а коэффичиенты $a_{j}$ определяются проинтегрированными коэффициентами Сили;

б) для любих вещественных $c_{1}, c_{2}$ и любого $\delta<\theta_{0}$ имеем

$$
\left|\mu^{-s} \Gamma\left(\frac{s}{2}\right) \zeta\left(\frac{s}{2}, \frac{D_{B}}{\mu^{2}}\right)\right| \leqslant C\left(c_{1}, c_{2}, \delta\right) e^{-\delta\left|\operatorname{Im} \frac{s}{2}\right|}, \quad\left|\operatorname{Im} \frac{s}{2}\right| \geqslant 1, \quad c_{1} \leqslant \operatorname{Re} \frac{s}{2} \leqslant c_{2} .
$$

Лемма 1 ясно показывает, что вакуумная энергия, вычисленная с помощью дзетарегуляризации (13), содержит сингулярность в виде полюса всегда, когда $a_{d+1} \neq 0$. Чтобы изучить поведение регуляризованного с помощью экспоненциального обрезания выражения (14), докажем следующую лемму.

ЛЕмма 2. При предположениях леммы 1 для функиии

$$
\frac{d h\left(t, \frac{D_{B}^{\frac{1}{2}}}{\mu}\right)}{d t}=\frac{d \operatorname{tr}\left(\exp \left\{-\frac{t}{\mu} D_{B}^{\frac{1}{2}}\right\}\right)}{d t}=\sum_{n}-\frac{\lambda_{n}^{\frac{1}{2}}}{\mu} \exp \left\{-t \frac{\lambda_{n}^{\frac{1}{2}}}{\mu}\right\}
$$


имеет место асимптотическое при $t \rightarrow 0$ разложение

$$
\begin{aligned}
\frac{d h\left(t, \frac{D_{B}^{\frac{1}{2}}}{\mu}\right)}{d t}=\sum_{k=0}^{d} & (-k) \frac{1}{2 \mu} \frac{\Gamma\left(\frac{k+1}{2}\right)}{\Gamma\left(\frac{1}{2}\right)} a_{d-k}\left(\frac{t}{2 \mu}\right)^{-k-1}+ \\
& +\sum_{k=1}^{K}(-k) \frac{1}{2 \mu} \frac{\Gamma\left(-k+\frac{1}{2}\right)}{\Gamma\left(\frac{1}{2}\right)} 2 a_{d+2 k}\left(\frac{t}{2 \mu}\right)^{2 k-1}+ \\
& +\sum_{k=0}^{K}(2 k+1) \frac{1}{2 \mu} \frac{(-1)^{k}}{\Gamma\left(\frac{1}{2}\right) \Gamma(k+1)}\left(\frac{t}{2 \mu}\right)^{2 k}\left[r_{d+2 k+1}\left(-k-\frac{1}{2}\right)+\right. \\
& +a_{d+2 k+1}\left(\Psi(1)+\sum_{l=0}^{k-1} \frac{1}{k-l}\right)+\sum_{j=0}^{d+2 k} \frac{2 a_{j}}{j-d-2 k-1}+ \\
& \left.+2 a_{d+2 k+1}\left((2 k+1) \ln \left(\frac{t}{2 \mu}\right)-\frac{1}{2 k+1}\right)\right]+\rho_{K}(t),
\end{aligned}
$$

$2 \partial e$

$$
\rho_{K}=O\left(\left(\frac{t}{2 \mu}\right)^{2 K+1+\varepsilon}\right), \quad 0<\varepsilon<1 .
$$

Отметим, что это асимптотическое разложение содержит при $t \rightarrow 0$ не только полюсы (в первой сумме в (16)), но также и логарифмические расходимости (при $k=0$ в последней сумме). Действительно, это разложение содержит полюсы порядка $d+$ $1, d, \ldots,-1$ с коэффициентами, определяемыми $a_{d-k}, k=0, \ldots, d$. Что касается коэффициента при логарифме, то он определяется $a_{d+1}$.

ДокАЗАТЕЛЬСтво ЛЕммы 2. Приведем здесь только набросок доказательства (детали см. в работе [13]). Для начала заметим, что

$$
\Gamma(s) \zeta\left(\frac{s}{2}, \frac{D_{B}}{\mu^{2}}\right)=\int_{0}^{\infty} t^{s-1} h\left(t, \frac{D_{B}^{\frac{1}{2}}}{\mu}\right) d t
$$

является преобразованием Меллина функции $h\left(t, D_{B}^{\frac{1}{2}} / \mu\right)$. Оно может быть также записано в виде

$$
\begin{aligned}
\Gamma(s) \zeta\left(\frac{s}{2}, \frac{D_{B}}{\mu^{2}}\right) & =\frac{\Gamma(s)}{\Gamma\left(\frac{s}{2}\right)}\left[\Gamma\left(\frac{s}{2}\right) \zeta\left(\frac{s}{2}, \frac{D_{B}}{\mu^{2}}\right)\right]= \\
& =\frac{2^{s-1}}{\sqrt{\pi}} \Gamma\left(\frac{s+1}{2}\right)\left[\Gamma\left(\frac{s}{2}\right) \zeta\left(\frac{s}{2}, \frac{D_{B}}{\mu^{2}}\right)\right]
\end{aligned}
$$

Из леммы 1 и известной структуры сингулярностей функции $\Gamma((s+1) / 2)$ следует, что выражение (17) аналитично при $\operatorname{Re} s>d$ и

$$
h\left(t, \frac{D_{B}}{\mu^{2}}\right)=\frac{1}{2 \pi i} \int_{c-i \infty}^{c+i \infty} d s\left(\frac{t}{\mu}\right)^{-s} \frac{2^{s-1}}{\sqrt{\pi}} \Gamma\left(\frac{s+1}{2}\right)\left[\mu^{-s} \Gamma\left(\frac{s}{2}\right) \zeta\left(\frac{s}{2}, \frac{D_{B}}{\mu^{2}}\right)\right],
$$


ВЫЧИСЛЕНИЕ ЭНЕРГИИ КАЗИМИРА С ПОМОЩЬЮ СПЕКТРАЛЬНЫХ ФУНКЦИЙ 113 где путь интегрирования таков, что $c>d$. Из (18) следует, что

$$
\begin{aligned}
\frac{d h\left(t, \frac{D_{B}}{\mu^{2}}\right)}{d t}= & \frac{1}{2 \pi i} \int_{c-i \infty}^{c+i \infty} d s \frac{(-s)}{\mu}\left(\frac{t}{\mu}\right)^{-s-1} \times \\
& \times \frac{2^{s-1}}{\sqrt{\pi}} \Gamma\left(\frac{s+1}{2}\right)\left[\mu^{-s} \Gamma\left(\frac{s}{2}\right) \zeta\left(\frac{s}{2}, \frac{D_{B}}{\mu^{2}}\right)\right],
\end{aligned}
$$

где интеграл берется вдоль того же контура, что и ранее.

Теперь, используя лемму 1 и тот факт, что

$$
\Gamma\left(\frac{s+1}{2}\right)=O\left(\exp \left\{\left(-\frac{\pi}{2}+\epsilon\right)\left|\operatorname{Im} \frac{s}{2}\right|\right\}\right)
$$

для любого $\epsilon>0$, можно получить асимптотическое разложение для $d h\left(t, D_{B} / \mu^{2}\right) / d t$, двигая контур интегрирования в (18) через полюсы

$$
\Gamma\left(\frac{s+1}{2}\right)\left[\mu^{-s} \Gamma\left(\frac{s}{2}\right) \zeta\left(\frac{s}{2}, \frac{D_{B}}{\mu^{2}}\right)\right]
$$

Эти полюсы находятся в точках $s=d-j$. Для $s=d-j=k \geqslant 0, j \leqslant d$, эти полюсы простые и дают следуюший вклад в интеграл Коши:

$$
\frac{-k}{2 \mu} \frac{\Gamma\left(\frac{k+1}{2}\right)}{\Gamma\left(\frac{1}{2}\right)} a_{d-k}\left(\frac{t}{2 \mu}\right)^{-k-1}, \quad k=0,1, \ldots, d
$$

Для $s=d-j=-2 k, k=1,2, \ldots$, полюсы также простые и их вклад равен

$$
-k \frac{1}{2 \mu} \frac{\Gamma\left(-k+\frac{1}{2}\right)}{\Gamma\left(\frac{1}{2}\right)} 2 a_{d+2 k}\left(\frac{t}{2 \mu}\right)^{2 k-1} .
$$

Для $s=d-j=-(2 k+1), k=0,1, \ldots$, полюсы простые и двойные. Они дают вклад

$$
\begin{aligned}
& \frac{2 k+1}{2 \mu} \frac{(-1)^{k}}{\Gamma\left(\frac{1}{2}\right) \Gamma(k)}\left(\frac{t}{2 \mu}\right)^{2 k}\left[r_{d+2 k+1}\left(-k-\frac{1}{2}\right)+\sum_{j=0}^{d+2 k} \frac{2 a_{j}}{j-d-2 k-1}\right] \times \\
& \quad \times \frac{2 k+1}{2 \mu} \frac{(-1)^{k}}{\Gamma\left(\frac{1}{2}\right) \Gamma(k+1)}\left(\frac{t}{2 \mu}\right)^{2 k} a_{d+2 k+1}\left[2 \ln \left(\frac{t}{2 \mu}\right)-2+\Psi(1)+\sum_{l=0}^{k-1} \frac{1}{k-l}\right],
\end{aligned}
$$


где сумму по $l$ нужно учитывать при $k>0$. Таким образом, двигая контур интегрирования в (18) до тех пор, пока не будет включена сингулярность $s=-(2 K+1)$, получим

$$
\begin{aligned}
\frac{d h\left(t, \frac{D_{B}}{\mu^{2}}\right)}{d t}= & \sum_{k=0}^{d}(-k) \frac{1}{2 \mu} \frac{\Gamma\left(\frac{k+1}{2}\right)}{\Gamma\left(\frac{1}{2}\right)} a_{d-k}\left(\frac{t}{2 \mu}\right)^{-k-1}+ \\
& +\sum_{k=1}^{K}(-k) \frac{1}{2 \mu} \frac{\Gamma\left(-k+\frac{1}{2}\right)}{\Gamma\left(\frac{1}{2}\right)} 2 a_{d+2 k}\left(\frac{t}{2 \mu}\right)^{2 k-1}+ \\
& +\sum_{k=0}^{K}(2 k+1) \frac{1}{2 \mu} \frac{(-1)^{k}}{\Gamma\left(\frac{1}{2}\right) \Gamma(k+1)}\left(\frac{t}{2 \mu}\right)^{2 k}\left[r_{d+2 k+1}\left(-k-\frac{1}{2}\right)+\right. \\
& +a_{d+2 k+1}\left(\Psi(1)+\sum_{l=0}^{k-1} \frac{1}{k-l}\right)+\sum_{j=0}^{d+2 k} \frac{2 a_{j}}{j-d-2 k-1}+ \\
& \left.+2 a_{d+2 k+1}\left((2 k+1) \ln \left(\frac{t}{2 \mu}\right)-\frac{1}{2 k+1}\right)\right]+\rho_{K}(t) .
\end{aligned}
$$

Остаток $\rho_{K}(t)$ дается таким же интегралом, как в $(19)$, но с $c<-2(K+1)$. Из утверждения "б" леммы 1 и уже обсуждавшейся оценки для $|\Gamma((s+1) / 2)|$ следует, что этот остаток есть $O\left(|t /(2 \mu)|^{2 K+1+\varepsilon}\right)$, что и завершает доказательство леммы 2 .

Полученное асимптотическое разложение при $t=0$ дает для экспоненциально регуляризованной вакуумной энергии (14) выражение

$$
\begin{aligned}
E_{\exp } & =-\left.\frac{\mu}{2} \frac{d h\left(t, \frac{D_{B}}{\mu^{2}}\right)}{d t}\right|_{t=0}=-\left.\frac{1}{2} \sum_{k=1}^{d}(-k) \frac{\Gamma\left(\frac{k+1}{2}\right)}{2^{-k} \Gamma\left(\frac{1}{2}\right)} a_{d-k}\left(\frac{t}{\mu}\right)^{-k-1}\right|_{t=0}- \\
- & \frac{1}{4 \Gamma\left(\frac{1}{2}\right)}\left[r_{d+1}\left(-\frac{1}{2}\right)+a_{d+1}(\Psi(1)-2)+2 \sum_{j=0}^{d} \frac{a_{j}}{j-d-1}\right]+\frac{1}{2} \frac{a_{d+1}}{\Gamma\left(\frac{1}{2}\right)} \ln \left(\frac{t}{2 \mu}\right) .
\end{aligned}
$$

Возврашаясь к лемме 1, для дзета-регуляризованной вакуумной энергии получаем

$$
\begin{aligned}
E_{\zeta}= & \left.\frac{\mu}{2} \zeta\left(\frac{s}{2}, \frac{D_{B}}{\mu^{2}}\right)\right|_{s=-1}=\frac{1}{2 \Gamma\left(-\frac{1}{2}\right)} \sum_{j=0}^{d} \frac{2 a_{j}}{j-d-1}+ \\
& +\frac{1}{2 \Gamma\left(-\frac{1}{2}\right)} r_{d+1}\left(-\frac{1}{2}\right)+\left.\frac{\mu^{s+1}}{\Gamma\left(\frac{s}{2}\right)} \frac{a_{d+1}}{s+1}\right|_{s=-1}= \\
= & -\frac{1}{2 \Gamma\left(\frac{1}{2}\right)} \sum_{j=0}^{d} \frac{a_{j}}{j-d-1}-\frac{1}{4 \Gamma\left(\frac{1}{2}\right)} r_{d+1}\left(-\frac{1}{2}\right)+ \\
& +\frac{1}{2 \Gamma\left(\frac{1}{2}\right)} a_{d+1}\left(\frac{\Psi(1)}{2}+1-\ln (2 \mu)\right)-\left.\frac{1}{2 \Gamma\left(\frac{1}{2}\right)} \frac{a_{d+1}}{s+1}\right|_{s=-1} .
\end{aligned}
$$

Из формул (21) и (22) можно сделать следуюшие выводы.

Оба метода регуляризации дают в общем случае расходяшиеся результаты. Следует различать два случая. 
СлучАй 1. Если $a_{d+1}=0$, дзета-регуляризация дает конечный результат, совпадающий с минимальной конечной частью экспоненциальной регуляризации. В последнем методе присутствуют полюсы порядков $2,3, \ldots, d+1$. Вычет в полюсе порядка $k+1$ равен произведению $\Gamma(k+1)$ на вычет $(\mu / 2) \zeta\left(s / 2, D_{B} / \mu^{2}\right)$ при $s=k, k=1, \ldots, d$.

СлучАй 2. В обшем случае $\left(a_{d+1} \neq 0\right)$ экспоненциальная регуляризация имеет кроме полюсов логарифмическую расходимость, коэффициент при которой равен минус вычету $(\mu / 2) \zeta\left(s / 2, D_{B} / \mu^{2}\right)$ при $s=-1$. Следовательно, разность между минимальными конечными частями в экспоненциальной и дзета-регуляризациях есть

$$
-\frac{1}{2} \frac{a_{d+1}}{\sqrt{\pi}} \Psi(1)=\frac{1}{2} \frac{a_{d+1}}{\sqrt{\pi}} \gamma,
$$

где $\gamma$ - константа Эйлера-Маскерони. Обе схемы регуляризации имеют логарифмическую зависимость от $\mu$ (см. [15] для случая дзета-регуляризации). Если разность между результатами двух регуляризаций состоит из ренормируемых членов, то возможна физическая интерпретация, и вся зависимость от $\mu$ исчезает.

Все вышеприведенные результаты верны также для случая многообразий без границы.

3.2. Массивное скалярное поле в $1+1$ измерениях. Теперь можно возвратиться к нашему примеру безмассового скалярного поля при $d+1=4$ и проверить, что оно соответствует случаю 1 из п. 3.1 .

В качестве простого примера случая 2 рассмотрим массивное скалярное полев $d+1=$ 2 измерениях, удовлетворяющее граничным условиям периодичности в пространственном направлении, $\varphi(t, L)=\varphi(t, 0)$. Легко видеть, что

$$
\omega_{n}=\left[m^{2}+\left(\frac{2 n \pi}{L}\right)^{2}\right]^{\frac{1}{2}}, \quad n \in \mathbb{Z} .
$$

Тогда с помощью дзета-регуляризации находим

$$
\begin{aligned}
E_{\zeta} & =\left.\frac{\mu}{2} \sum_{n=-\infty}^{\infty}\left[\left(\frac{2 n \pi}{L \mu}\right)^{2}+\left(\frac{m}{\mu}\right)^{2}\right]^{-\frac{s}{2}}\right|_{s=-1}= \\
& =\left.\frac{\mu^{s+1}}{2}\left(\frac{2 \pi}{L}\right)^{-s} \sum_{n=-\infty}^{\infty}\left[n^{2}+\left(\frac{m L}{2 \pi}\right)^{2}\right]^{-\frac{s}{2}}\right|_{s=-1}
\end{aligned}
$$

Используя представление (3), это выражение можно переписать в виде

$$
\begin{gathered}
\left.\frac{\mu^{s+1}}{2}\left(\frac{2 \pi}{L}\right)^{-s} \sum_{n=-\infty}^{\infty} \frac{1}{\Gamma\left(\frac{s}{2}\right)} \int_{0}^{\infty} d t t^{\frac{s}{2}-1} \exp \left\{-\left(n^{2}+\left(\frac{m L}{2 \pi}\right)^{2}\right) t\right\}\right|_{s=-1}= \\
=\left.\frac{\mu^{s+1}}{2}\left(\frac{2 \pi}{L}\right)^{-s} \frac{1}{\Gamma\left(\frac{s}{2}\right)} \int_{0}^{\infty} d t t^{\frac{s}{2}-1} \exp \left\{-\left(\frac{m L}{2 \pi}\right)^{2} t\right\} \Theta\left(0, \frac{t}{\pi}\right)\right|_{s=-1},
\end{gathered}
$$


где

$$
\Theta(x, y)=\sum_{n=-\infty}^{\infty} e^{2 \pi x n-\pi y n^{2}}
$$

есть тЭта-функция Якоби, для которой выполняется следуюшее свойство инверсии:

$$
\Theta(x, y)=\frac{1}{\sqrt{y}} e^{\frac{\pi x^{2}}{y}} \Theta\left(\frac{x}{i y}, \frac{1}{y}\right) .
$$

Используя это свойство, можно показать, что

$$
\begin{aligned}
E_{\zeta}= & \frac{\mu^{s+1}}{2}\left(\frac{2 \pi}{L}\right)^{-s} \frac{1}{\Gamma\left(\frac{s}{2}\right)}\left[\left(\frac{2 \pi}{m L}\right)^{s-1} \Gamma\left(\frac{s-1}{2}\right)+\right. \\
& \left.+2 \int_{0}^{\infty} d t t^{\frac{s-1}{2}-1} \exp \left\{-\left(\frac{m L}{2 \pi}\right)^{2} t\right\} \exp \left\{-\frac{n^{2} \pi^{2}}{t}\right\}\right]\left.\right|_{s=-1} .
\end{aligned}
$$

Отсюда можно увидеть, что полюс при $s=-1$ обусловлен полюсом гамма-функции. Вычисляя интеграл и разлагая вблизи $s=-1$, получаем

$$
E_{\zeta}^{(1)}=-\frac{m}{\pi} \sum_{n=1}^{\infty} \frac{1}{n} K_{1}(n m L)+\frac{m^{2} L}{4 \pi}\left[\left.\frac{1}{s+1}\right|_{s=-1}-\ln \left(\frac{m}{2 \mu}\right)-\frac{1}{2}\right] .
$$

Ряд в (24) сходится благодаря свойствам модифицированных функций Бесселя $K_{1}$ при $n \rightarrow \infty$.

Регуляризация с помощью экспоненциального обрезания дает

$$
E_{\exp }^{(1)}=-\left.\frac{\mu}{2} \frac{d}{d t}\left(\sum_{n=-\infty}^{\infty} \exp \left\{-t\left(\left(\frac{2 n \pi}{L \mu}\right)^{2}+\left(\frac{m}{\mu}\right)^{2}\right)^{\frac{1}{2}}\right\}\right)\right|_{t=0} .
$$

Этот ряд может быть переписан с использованием формулы суммирования Пуассона

где

$$
\sum_{n=-\infty}^{\infty} f(n)=\sum_{p=-\infty}^{\infty} c_{p}
$$

$$
c_{p}=\int_{-\infty}^{\infty} d x e^{2 \pi i p x} f(x)
$$

Вычислив производную и положив $t=0$, получаем

$$
E_{\exp }^{(1)}=-\frac{m}{\pi} \sum_{n=1}^{\infty} \frac{1}{n} K_{1}(n m L)+\left.\frac{m^{2} L}{4 \pi}\left[-\ln t-\ln \left(\frac{m}{2 \mu}\right)+2\left(\frac{m t}{\mu}\right)^{-2}-\gamma-\frac{1}{2}\right]\right|_{t=0} .
$$

Этот результат также расходится. Как и предсказьвалось, возникают расходимости в виде полюсов и логарифмов. Сравнение коэффициентов данного выражения с коэффициентами в выражении (24) показывает полное соответствие с нашим общим результатом.

Оба результата допускают перенормировку с требованием $E \rightarrow 0$ при $R \rightarrow \infty$ или, что эквивалентно, $E \rightarrow 0$ при $m \rightarrow \infty$ (как предложено в работе [16]). При этом получается физически осмысленный результат и отсутствует зависимость от $\mu$.

Открытым остается вопрос, что происходит в более сложных геометриях, в частности при наличии искривленных границ. 
Благодарности. Данная работа частично поддержана АNPCyT (PICT'97 00039), CONICET(PIP 0459) и UNLP (Proy. 11-X230), Argentina.

Я благодарна К. Ж. Беневентано, М. де Франсиа и Х. Фаломиру за многочисленные полезные комментарии и предложения, касаюшиеся изложения материала данной статьи. Я также благодарна Д. В. Василевичу и организаторам Международной школы физики им. В. А. Фока за предоставленную возможность участия в Школе. Особая благодарность Юрию и Виктору Новожиловым и Валерию Марачевскому за их тепльй прием.

\section{Список литературы}

[1] К. Ициксон, Ж.-Б. Зюбер. Квантовая теория поля. М.: Мир, 1984.

[2] H. B. G. Casimir. Proc. K. Ned. Akad. Wetensc. 1948. V. 51. P. 793.

[3] G. Plunien, B. Muller, W. Greiner. Phys. Rep. 1986. V. 134. P. 87.

[4] V. M. Mostepanenko, N. N. Trunov. The Casimir Effect and its Applications. Oxford: Clarendon Press, 1997.

[5] J. S. Dowker, R. Critchley. Phys. Rev. D. 1976. V. 13. P. 3224.

[6] S. W. Hawking. Commun. Math. Phys. 1977. V. 55. P. 133.

[7] М. А. Абрамович, И. А. Стиган. Справочник по специальным функциям с формулами, графиками и математическими таблицами. М.: Мир, 1979.

[8] R. T. Seeley. AMS Proc. Symp. Pure Math. 1967. V. 10. P. 288.

[9] R. T. Seeley. Am. J. Math. 1969. V. 91. P. 889.

[10] R. T. Seeley. Am. J. Math. 1969. V. 91. P. 963.

[11] S. Agmon. Commun. Pure Appl. Math. 1967. V. 10. P. 288.

[12] P. B. Gilkey. Invariance Theory, the Heat Equation, and the Atiyah-Singer Index Theorem. Vilmington: Publish or Perish, 1984.

[13] C. G. Beneventano, E. M. Santangelo. Int. J. Mod. Phys. A. 1996. V. 11. P. 2871.

[14] G. Grubb, R. T. Seeley. J. Geom. Anal. 1996. V. 6. P. 31.

[15] S. Blau, M. Visser, A. Wipf. Nucl. Phys. B. 1988. V. 310. P. 163.

[16] M. Bordag, K. Kirsten. Phys. Rev. D. 1999. V. 60. P. 105019. 